\title{
Fine Mapping of Intracranial Aneurysm Susceptibility Based on a Genome-wide Association Study
}

\author{
Eun Pyo Hong \\ Hallym University College of Medicine \\ Dong Hyuk Youn \\ Hallym University College of Medicine \\ Bong Jun Kim \\ Hallym University College of Medicine \\ Jun Hyong Ahn \\ Hallym University College of Medicine \\ Jeong Jin Park \\ Konkuk Medical Center \\ Jong Kook Rhim \\ Jeju National University College of Medicine

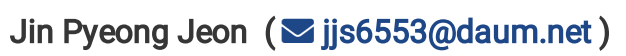 \\ Hallym University College of Medicine
}

\section{Research Article}

Keywords: Fine mapping, Genome-wide association study, Intracranial aneurysm, Variant causality

Posted Date: August 9th, 2021

DOI: https://doi.org/10.21203/rs.3.rs-777954/v1

License: (1) This work is licensed under a Creative Commons Attribution 4.0 International License. Read Full License

Version of Record: A version of this preprint was published at Scientific Reports on February 17th, 2022. See the published version at https://doi.org/10.1038/s41598-022-06755-x. 


\section{Abstract}

In addition to conventional genome-wide association studies (GWAS), a fine-mapping is increasingly used to identify the genetic function of variants associated with disease susceptibilities. Here, we used a fine-mapping approach to evaluate the casual variants based on a previous GWAS involving patients with intracranial aneurysm (IA). Fine-mapping analysis was conducted based on the chromosomal data provided by GWAS consisting 250 patients diagnosed with IA and 296 controls using posterior inclusion probability (PIP) and log10 transformed Bayes factor (log10BF). The narrow sense of heritability $\left(h^{2}\right)$ explained by each casual variant was estimated. Subsequent gene expression and functional network analyses were used to calculate the transcripts per million (TPM) values. Twenty causal candidate single nucleotide polymorphisms (SNPS) surpassed a genome-wide significance threshold for creditable evidence (log10BF > 6.1). Four SNPs including rs75822236 (R535H, GBA; log10BF = 15.06), rs112859779 (G141S, TCF24; $\log 10 \mathrm{BF}=12.12)$, rs79134766 (A208T, OLFML2A; log10BF = 14.92), and rs371331393 (Q1932X, ARHGAP32; log10BF = 20.88) showed a completed PIP value in each chromosomal region, suggesting a high probability of variant causality associated with IA. Expression in GBA was highly enriched in the whole blood (TPM $=33.13$ ), while TCF24 were rarely expressed in all tissues and cells. No direct interaction was observed between the four casual genes; however, $P S A P$ appeared to be particularly important via indirect correlation between other genes. Our results suggested that four mutations of GBA, TCF24, OLFML2A, and ARHGAP32 were linked to IA susceptibility and pathogenesis. Our approach may promise more informative mutations in the following GWAS.

\section{Introduction}

Intracranial aneurysm (IA) refers to an abnormal focal dilatation of a cerebral artery due to a weakening of the intima of a blood vessel wall. The prevalence of IA in the general adult population has been reported to be nearly $6 \%^{1}$. The incidence of unruptured intracranial aneurysms (UIAs) was 15.6 per 100,000 persons ${ }^{2}$. The asymptomatic UIA can rupture suddenly resulting in subarachnoid hemorrhage (SAH), which is associated with a higher mortality rate exceeding $50 \%$ within one month after ictus ${ }^{3,4}$. IA is a complex disease involving an interaction between clinical and genetic factors underlying its formation and growth ${ }^{2}$. Important clinical risk factors for IA include female gender, hypertension and smoking. The risk of rupture is increased when the aneurysm is located between arterial branches or in the vertebrobasilar region, in addition to larger size at diagnosis, and the presence of a bleb or daughter sac ${ }^{5-7}$. Genetic studies have been performed to identify genes associated with IA via linkage analysis and single nucleotide polymorphisms (SNPs) of known candidate genes, strongly correlated genes or genome-wide association studies (GWAS) for screening multiple candidate genes. In particular, GWAS revealed large-scale genetic associations, which were primarily correlated with traits and diseases. GWAS technically compares allele frequencies in SNPs between cases and controls. However, complex traits of IA are not entirely attributed to a single gene, but are caused by the influence of multiple genes ${ }^{8,9}$. Gene-gene and gene-environment interactions also affect the traits and diseases. Given the inherent features of the GWAS, genetic markers included in the same linkage disequilibrium exhibit similar correlation. Accordingly, even if a candidate gene is identified via GWAS, it could merely suggest a statistically significant difference rather than represent an etiological factor. Further, the precise location of the causative gene may differ in the same linkage disequilibrium (LD) block. Thus, it is important to reduce the errors via additional data processing to identify false-positive results obtained in GWAS.

Fine-mapping is one of the post-GWAS analyses used to narrow potential candidate variants directly affecting the trait ${ }^{10}$. This approach can be used to identify the regions associated with possible causal susceptibility based on the population of structures with LD $^{9}$. It provides a complex correlation between the casual variants and the disease development using computational data without in vivo and in vitro molecular biology studies. ${ }^{10,11}$ Sekar et al. ${ }^{11}$ showed that structurally diverse alleles of the complement component 4 genes contribute to schizophrenia via excessive complement activity, resulting in reduced numbers of synapses. Consequently, it can be used to assess the functional role of the risk allele, which is a challenge to investigate based on molecular mechanisms, despite the strong genetic association. Fine-mapping of complex traits has been increasingly performed in many diseases, especially cancer and stroke, but has yet to be reported in IA. Here, for the first time, we performed a fine-mapping analysis based on previous GWAS data sets to identify the causal candidate variants in an effort to identify the precise genetic variants associated with IA in a Korean adult cohort. We also performed a functional gene set enrichment analysis using the optimized candidate sets to analyze the biological relationship between candidate genes and IA.

\section{Materials And Methods}

\section{GWAS-based summary statistics}


The analysis was based on the summary statistics provided by the previous IA GWAS. In brief, the study included 250 adult patients with saccular aneurysm and 296 controls between March 2015 and December 202012,13. The AxiomTH Asian Precision Medicine Research Array (APMRA) (Thermo Fisher Scientific, MA, USA) were used for genotyping of the study subjects. High-quality plates were defined by a plate pass rate higher than $95 \%$ for samples. The average call rate of passing samples was greater than $99 \%$. A total of 512,575 SNPs passed the quality control including genotyping call rate of $95 \%$ or higher, minor allele frequency of at least $1 \%$, and Hardy-Weinberg equilibrium $P$-value $\geq 1 \times 10^{-612}$. GWAS-based summary statistics included allele types, minor allele frequencies, effect sizes, and p-values. The Institutional Review Board and Ethics Committee approved all protocols of the study (No. 2016-3, 2019-06-006).

\section{Statistical Analysis}

We performed a fine-mapping study to identify the role of casual candidate combinations in the susceptibility to IA using FINEMAP v.1.3.1 ${ }^{14}$. Odds ratios (ORs) of individual SNPs were converted using the natural log-transformed formula (InOR). Causality of each SNP or configuration was assessed using effect sizes, posterior inclusion probabilities (PIPs), and narrow sense of heritability (h2), which was explained by casual SNPs. The log10-transformed Bayes factors (log10BF) for the individual casual SNPs and configurations were estimated via FINEMAP analysis. A log10BF value greater than 6.1 suggested significant genome-wide evidence. The fine-mapping approach requires the estimates of SNP correlations, and therefore LD matrices between SNPs were generated by PLINK v1.9 (https://www.cog-genomics.org/plink/) ${ }^{15}$. All the fine-mapping tests were conducted with individual chromosomes (chr1-22) due to the LD-based mapping procedure. Manhattan and regional association plots of fine-mapping results were obtained using the package of "qqman" in R v3.6.1 (https://cran.r-project.org/web/packages/qqman) and LocusZoom v1.3 written in the modified Python and R scripts ${ }^{16}$. Regional annotations and functional impact of SNPs were described using the ANNOVAR program including the PolyPhen-2 (http://www.openbioinformatics.org/annovar/) ${ }^{17}$.

\section{Gene expression and functional network analyses}

The expressions of causal candidate genes was evaluated in human blood, brain-specific tissues, or cells using the Genotype-Tissue Expression (GTEx) Portal (https://gtexportal.org/home/) ${ }^{18}$. Transcripts per million (TPM) values of a total of 56,200 genes were calculated in 13 brain tissues, 4 blood vessels ( 3 arterial tissues and 1 cell line of EBV-transformed lymphocytes), and a whole blood cell. Subsequent gene functional network analysis was conducted using the GeneMANIA program (https://genemania.org/) ${ }^{19}$.

\section{Results}

\section{Fine-mapping analyses}

Fine mapping analyses entailed multiple SNP combinations by each autosome chromosome. A total of 20 causal candidate SNPS surpassed a genome-wide significance threshold for creditable evidence $(\log 10 \mathrm{BF}>6.1)$ (Fig. 1a). The log10BF values were strongly correlated with GWAS-based $p$-values $\left(R^{2}\right.$ for correlation $\left.=0.9861, \mathrm{p}<0.0001\right)$ (Fig. 1b). Among them, 13 SNPs showed a high PIP in each chromosomal region (PIP>0.8) (Fig. 1c). The PIPs were slightly correlated with GWAS-driven p-values $\left(R^{2}\right.$ for correlation $=0.0974$, $p<0.0001$ ) (Fig. 1D). Six out of 13 SNPs were located on exonic regions (Table 1). The rs371331393 (Q1932X), one SNP of ARHGAP32 (11q24.3), exhibited a stop-gain function and was most significantly associated with increased IA risk ( $\operatorname{lnOR}=3.77, \log 10 \mathrm{BF}=20.88$ ). This variant showed the highest heritability $\left(h^{2}=0.143\right)$. Three SNPs including rs75822236 (R535H, $\left.G B A ; \log 10 \mathrm{BF}=15.06\right)$, rs112859779 (G141S, TCF24; $\log 10 \mathrm{BF}=12.12$ ), and rs79134766 (A208T, OLFML2A; log10BF =14.92) were associated with amino acid substitutions, which may contribute to possible DNA sequence damage and increased association with IA. Eighteen SNPs, including PRDM2, FMO4, and RNF144A, which exceeded genome-wide significance level in GWAS $\left(p<5 \times 10^{-8}\right)$, were associated with low causality to IA formation $\left(1.89 \times 10^{-11}<\mathrm{PIP}<0.13\right.$, log10BF $\left.>5\right)$ (Supplementary Table 1). In summary, fine mapping annotated that $A R H G A P, G B A$, $T C F 24$, and OLFML2A would be casual candidate genes. 
Table 1

Significant causal susceptibility identified by fine-mapping after genome-wide association study

\begin{tabular}{|c|c|c|c|c|c|c|c|c|c|c|}
\hline Gene & $\mathrm{Chr}$ & Function & SNP & $M / m^{a}$ & MAF & PIPb & $\log 10 \mathrm{BF}$ & $h^{2 b}$ & InOR & $\begin{array}{l}P \text { in } \\
\text { GWAS }\end{array}$ \\
\hline PRDM2 & $1 \mathrm{p} 36.21$ & intronic & rs61775135 & $\mathrm{C} / \mathrm{A}$ & 0.285 & $\begin{array}{l}2.61 \mathrm{E}- \\
06\end{array}$ & 9.48 & 0.0733 & -1.15 & $\begin{array}{l}3.59 \mathrm{E}- \\
13\end{array}$ \\
\hline$G B A$ & $1 q 22$ & $\begin{array}{l}\mathrm{R} 535 \mathrm{H}, \\
\text { exon11 }\end{array}$ & rs75822236 & $\mathrm{C} / \mathrm{T}$ & 0.166 & 1.0000 & 15.06 & 0.1088 & 5.08 & $\begin{array}{l}1.09 \mathrm{E}- \\
19\end{array}$ \\
\hline FMO4 & $1 q 24.3$ & $\begin{array}{l}F 281 F \\
\text { exon8 }\end{array}$ & rs3737926 & $\mathrm{C} / \mathrm{T}$ & 0.264 & $\begin{array}{l}1.10 \mathrm{E}- \\
08\end{array}$ & 7.10 & 0.0577 & -1.01 & $\begin{array}{l}1.83 \mathrm{E}- \\
10\end{array}$ \\
\hline$R N F 144 A$ & $2 p 25.1$ & intron & rs6741819 & $\mathrm{C} / \mathrm{T}$ & 0.247 & 0.0905 & 10.30 & 0.0785 & -1.38 & $\begin{array}{l}4.05 \mathrm{E}- \\
14\end{array}$ \\
\hline HDAC4,LOC150935 & $2 q 37.3$ & intergenic & rs59626274 & $\mathrm{C} / \mathrm{T}$ & 0.248 & 0.0679 & 10.18 & 0.0777 & -1.34 & $\begin{array}{l}5.78 \mathrm{E}- \\
14\end{array}$ \\
\hline LINC01237 & $2 q 37.3$ & $\begin{array}{l}\text { ncRNA, } \\
\text { intron }\end{array}$ & rs78458145 & $\mathrm{G} / \mathrm{A}$ & 0.285 & 0.8416 & 11.27 & 0.0847 & -1.41 & $\begin{array}{l}3.14 \mathrm{E}- \\
15\end{array}$ \\
\hline SPCS3,VEGFC & $4 q 34.2$ & intergenic & rs17688188 & $\mathrm{G} / \mathrm{A}$ & 0.222 & 0.9999 & 9.29 & 0.0718 & -1.31 & $\begin{array}{l}5.99 \mathrm{E}- \\
13\end{array}$ \\
\hline TCF24 & $8 q 13.1$ & $\begin{array}{l}\text { G141S, } \\
\text { exon4 }\end{array}$ & rs112859779 & $\mathrm{C} / \mathrm{T}$ & 0.216 & 1.0000 & 12.12 & 0.0898 & -1.69 & $\begin{array}{l}3.33 \mathrm{E}- \\
16\end{array}$ \\
\hline$O L F M L 2 A$ & $9 q 33.3$ & $\begin{array}{l}\text { A208T, } \\
\text { exon4 }\end{array}$ & rs79134766 & $\mathrm{G} / \mathrm{A}$ & 0.219 & 1.0000 & 14.92 & 0.1072 & -1.97 & $\begin{array}{l}1.70 \mathrm{E}- \\
19\end{array}$ \\
\hline MYEOV,LINC01488 & $11 q 13.3$ & intergenic & rs76855873 & $\mathrm{C} / \mathrm{T}$ & 0.269 & $\begin{array}{l}1.89 \mathrm{E}- \\
13\end{array}$ & 8.15 & 0.0642 & -1.11 & $\begin{array}{l}1.23 \mathrm{E}- \\
11\end{array}$ \\
\hline ARHGAP32 & $11 q 24.3$ & $\begin{array}{l}\text { Q1932X, } \\
\text { exon22 }\end{array}$ & rs371331393 & $\mathrm{G} / \mathrm{A}$ & 0.171 & 1.0000 & 20.88 & 0.1435 & 3.77 & $\begin{array}{l}9.32 \mathrm{E}- \\
27\end{array}$ \\
\hline CD163L 1 & 12p13.31 & splicing & rs138525217 & $\mathrm{C} / \mathrm{T}$ & 0.161 & 1.0000 & 17.77 & 0.1248 & 4.33 & $\begin{array}{l}6.20 \mathrm{E}- \\
23\end{array}$ \\
\hline$S L C 2 A 14$ & 12p13.31 & intron & rs118107419 & $\mathrm{C} / \mathrm{A}$ & 0.262 & $\begin{array}{l}8.60 \mathrm{E}- \\
08\end{array}$ & 10.71 & 0.0807 & -1.40 & $\begin{array}{l}1.44 \mathrm{E}- \\
14\end{array}$ \\
\hline CUL $4 A, L A M P 1$ & $13 q 34$ & intergenic & rs74115822 & $\mathrm{G} / \mathrm{A}$ & 0.112 & 0.9960 & 7.32 & 0.0584 & 1.83 & $\begin{array}{l}1.12 \mathrm{E}- \\
10\end{array}$ \\
\hline MIR365A,PARN & $16 p 13.12$ & intergenic & rs11646803 & $\mathrm{C} / \mathrm{T}$ & 0.376 & 0.9422 & 6.77 & 0.0548 & -0.79 & $\begin{array}{l}4.76 \mathrm{E}- \\
10\end{array}$ \\
\hline MINK1 & 17p13.2 & intron & rs72835045 & $\mathrm{G} / \mathrm{A}$ & 0.220 & 0.9984 & 8.92 & 0.0688 & -1.38 & $\begin{array}{l}1.69 \mathrm{E}- \\
12\end{array}$ \\
\hline NAPA-AS1 & $19 q 13.32$ & $\begin{array}{l}\text { ncRNA, } \\
\text { intron }\end{array}$ & rs55800589 & $\mathrm{G} / \mathrm{C}$ & 0.364 & 1.0000 & 9.53 & 0.0726 & -0.96 & $\begin{array}{l}3.35 \mathrm{E}- \\
13\end{array}$ \\
\hline DSCAM & $21 q 22.2$ & intron & rs727333 & $\mathrm{C} / \mathrm{A}$ & 0.257 & 0.1247 & 10.38 & 0.0773 & -1.36 & $\begin{array}{l}3.69 \mathrm{E}- \\
14\end{array}$ \\
\hline$\angle R R C 3$ & $21 q 22.3$ & $\begin{array}{l}\text { P63P, } \\
\text { exon2 }\end{array}$ & rs116969723 & $\mathrm{G} / \mathrm{A}$ & 0.233 & 0.8753 & 11.23 & 0.0827 & -1.45 & $\begin{array}{l}3.83 \mathrm{E}- \\
15\end{array}$ \\
\hline
\end{tabular}

GWAS, genome-wide association study; $\log 10 \mathrm{BF}$, $\log 10$ transformed Bayes factor; InOR, natural log-transformed odds ratio; PIP, posterior inclusion probability; MAF, minor allele frequency.

${ }^{\text {a }} \mathrm{M} / \mathrm{m}$ indicates major/minor allele type, respectively.

${ }^{\mathrm{b}} \mathrm{PIP}, \log 10 \mathrm{BF}$, and heritability $\left(h^{2}\right)$ of individual variants were estimated via FINEMAP program to identify causal susceptibility to intracranial aneurysm (IA).

${ }^{\mathrm{C}}$ InOR and $P$-value were estimated by IA GWAS. 


\begin{tabular}{|c|c|c|c|c|c|c|c|c|c|c|}
\hline Gene & Chr & Function & SNP & $\mathrm{M} / \mathrm{m}^{\mathrm{a}}$ & MAF & PIPb & $\log 10 \mathrm{BF}$ & $h^{2 b}$ & InOR & $\begin{array}{l}P \text { in } \\
\text { GWAS }\end{array}$ \\
\hline RFPL2,SLC5A4 & $22 q 12.3$ & intergenic & rs117398778 & $\mathrm{T} / \mathrm{C}$ & 0.138 & 0.9397 & 6.24 & 0.0506 & 1.32 & $\begin{array}{l}2.00 \mathrm{E}- \\
09\end{array}$ \\
\hline
\end{tabular}

GWAS, genome-wide association study; log10BF, log10 transformed Bayes factor; InOR, natural log-transformed odds ratio; PIP, posterior inclusion probability; MAF, minor allele frequency.

${ }^{a} \mathrm{M} / \mathrm{m}$ indicates major/minor allele type, respectively.

b PIP, $\log 10 \mathrm{BF}$, and heritability $\left(h^{2}\right)$ of individual variants were estimated via FINEMAP program to identify causal susceptibility to intracranial aneurysm (IA).

${ }^{\mathrm{C}}$ InOR and $P$-value were estimated by IA GWAS.

\section{Gene expression and functional network analyses}

Gene expression and functional network analyses were performed using the four causal candidate genes (Fig. 2 and Supplementary Table 2). GBA was broadly enriched in all tissues and cell lines $(6<\mathrm{TPM}<34)$. In particular, it was highly expressed in the whole blood (TPM $=33.13)$. Conversely, TCF24 was rarely expressed in all tissues and cells $(T P M<0.15)$. Expression in $O L F M L 2 A$ was moderate in all arteries (TPM $=2.37$ to 9.54). ARHGAP32 was rarely enriched in EBV-transformed lymphocytes and whole blood (TPM = 0.0667 and 0.2274 , respectively), while it was enriched in the brain and blood vessels (TPM, between 3 and 24). No direct interaction was observed between the four casual genes (Fig. 3). However, these genes constitute a hub network interacting with neighbor genes, especially PSAP, SCARB2, and ASAH1 (Fig. 3).

\section{Discussion}

Large-scale GWAS and meta-analyses have been widely used to identify and validate common or novel susceptible gene variants in various medical diseases over the past decade. However, given the overall genotype-phenotype analyses, disease-modifying functional mutations and direct biological relevance to disease have yet to be elucidated completely ${ }^{20}$. In addition, the heritability of a specific trait cannot be fully explained by common SNPs of intronic or intergenic regions via GWASs that have been targeted to identify common variants in common complex diseases. Accordingly, even if a large number of susceptible loci were identified, a few cases showed their replication in an independent cohort. Thus, few disease-associated variants have been demonstrated in functional in vitro studies or used in treatment ${ }^{21}$. To overcome these limitations, an updated fine-mapping analysis was performed to identify the variant causality associated with human complex diseases and as a cost-effective genotyping strategy ${ }^{9}$. To date, many studies underscored the need for 'feature selection' to identify relevant "variables" using parametric or non-parametric models. However, feature selection is not a simple challenge and requires substantial genetic investigations. It is important to identify the causal or driver mutations linked to treatment of human complex diseases. The selection of genetic variants from GWAS is uncertain given the strongly correlated SNPs corresponding to a pairwise LD structure at the population level. A fine-mapping analysis facilitates the identification of creditable genetic variants to refine the selection bias such as false-positive variants based on the initial GWAS and to improve the findings of molecular functional studies $^{9}$. Here, we performed a fine-mapping analysis based on the results of previous IA GWAS using the statistical method developed by Benner et al. ${ }^{14}$ Our findings may enable the identification of causal variants (true negative) and exclude potential false positives via statistically significant fine-mapping analysis of transformed GWAS results. Therefore, these analytical methods may enable the selection of functional candidate variants based on the molecular mechanisms associated with IA formation.

In this study, we found four causal genes that are potentially linked to IA such as GBA, TCF24, OLFML2A, and ARHGAP32. We speculated that these genetic variants may cause dysfunctional immune response and inflammation in DNA sequences damaged by amino acid substitution or gain- or loss-of-function mutations, which affects the IA formation. GBA located in the exonic regions of 1q22 (rs75822236) was significantly associated with $\mathrm{IA}^{12}$. More specifically, GWAS revealed that the " $\mathrm{T}$ " allele of this variant increased the risk of IA. ${ }^{12}$ In addition, a fine-mapping analysis also revealed a higher level of log10BF (15.06) and PIP (1.0), suggesting that this variant was a true positive for IA. The role of GBA was mainly investigated in Parkinson's disease (PD) or Gaucher disease (GD), which is a recessive lysosomal storage disorder, and barely investigated in IA. Mata et al. ${ }^{22}$ reported that $G B A$ mutations and E326K carrier were related to impaired working memory and executive function in patients with $\mathrm{PD}$. In GD, null or severe homozygous mutations of $G B A$ showed little or no human glucocerebrosidase activity ${ }^{23}$. These findings suggested differences in phenotype due to the various $G B A$ 
mutations. Kleinloog et al. ${ }^{24}$ reported enrichment of the lysosomal pathway in ruptured IA compared with UIA based on RNA sequencing analysis of aneurysm wall. Although the lysosomal pathway does not reflect an acute reaction to IA rupture ${ }^{24}$, it is likely that it is induced by inflammation after bleeding.

OLFML2A and TCF24 showed a protective effect against IA formation with log10BF levels greater than 12 and completed PIPs. However, the relationship between these two genes and IA is still unclear, even though it has been implicated in cardiovascular diseases. Conversely, ARHGAP32 significantly increased the risk of IA with the highest log10BF (20.88) and completed PIP. ARHGAP32 refers to Rho GTPase-activating protein 32 and mediates N-methyl D-aspartate receptor signaling ${ }^{12}$. The role of $A R H G A P 32$ has been mainly investigated in the regulation of blood pressure. Rho-specific GTPase-activating protein GRAF3 was highly expressed in smooth muscle cells (SMCs) and regulated blood pressure control by inhibiting the contractility of RhoA-mediated SMC ${ }^{25}$. GRAF3-deficient mice also showed increased blood pressure in response to angiotensin II and endothelin $1^{26}$. In actual clinical practice, many patients manifest both IA and hypertension. Inci et al. ${ }^{27}$ reported that the rate of pre-existing hypertension was $43.5 \%$ in patients with IA, which was higher than $24.4 \%$ in the normal population. Hypertension may contribute to degeneration of the internal elastic lamina, weakening of the vessel wall, and IA formation ${ }^{27}$. Nevertheless, it is unclear whether the role of ARHGAP32 in IA is mediated indirectly via chronic hypertension or directly via change in vascular tone.

Functional network analyses showed that PSAP was an important gene in the development of IA. The role of PSAP gene was rarely investigated in IA and was mainly studied in PD. Oji et al. ${ }^{28}$ reported that two SNPs of rs4747203 and rs885828, the intronic regions of the PSAP saposin D domain were linked to PD. PSAP mutation can also result in dopaminergic neurodegeneration and motor decline in mice. Although we did not include patients with PD, a fine-mapping analysis revealed that PD-related genes such as GBA and $P S A P$ may contribute to IA. Lysosomal dysfunction and the resulting lysosomal storage disorder can contribute causally to PD. Putative damaging variants in at least one gene associated with lysosomal storage disorder were observed in most PD patients ${ }^{29}$. However, lysosomal dysfunction can also be observed in the arterial wall. Lysosomal changes in the vascular SMCs were attributed to the accumulation of excessive substrate levels in the lysosomes of a primate model of atherosclerosis and hypertension ${ }^{30}$. The excessive sterol accumulation in lysosomes can disrupt the lysosomal function ${ }^{31}$. Therefore, in this case, it is possible that lysosomal dysfunction may directly affect IA formation or may contribute to IA via atherosclerosis. Hokari et al. ${ }^{32}$ reported that atherosclerotic factors strongly increased the risk of middle cerebral artery aneurysm compared with paraclinoid aneurysm. After securing aneurysm, consistent statin therapy was significantly correlated with better prognosis. ${ }^{33}$ Wu et al. ${ }^{34}$ demonstrated that the autophagy-lysosomal pathway, which entails self-digestion of dysfunctional intracellular components by lysosomal enzymes, was an important pro-survival mechanism after SAH. However, the study investigated the role of the lysosome after SAH development, but not in IA development itself. Therefore, additional studies are needed to investigate the role of candidate variants in lysosomal dysfunction resulting in IA formation via abnormal ECM remodeling in response to hemodynamic stress.

A fine-mapping analysis may enable the detection of mutations involving the candidate genes and thereby contribute to treatment based on genome-based precision medicine. Identifying the causal variants beyond GWAS provides a crucial blueprint in predicting disease risk and preventing IA as well as metabolic diseases and stroke ${ }^{35}$. To the best of our knowledge, this was the first fine-mapping analysis based on the results of a GWAS of IA. Nevertheless, the interpretation of the results may have some limitations. First, the sample size was relatively somehow small to discovery more functional mutations to achieve a sufficient statistical power, even though four common mutations have been detected in our study. Second, variables regarding aneurysm location and size were not considered in the analysis. Microscopic analysis of the aneurysm during actual surgery often reveals atherosclerotic changes in the aneurysm wall, especially in elderly patients with larger aneurysm. Accordingly, GWAS and subsequent fine-mapping analysis should be performed together given the metabolic status of a large number of patients with IA.

In summary, fine-mapping analysis robustly identified four functional mutations of causal candidate genes, such as GBA, TCF24, $O L F M L 2 A$, and ARHGAP32 that have associated with IA. Mutations in these genes show play roles in immune and inflammatory systems according to our literature review and functional annotations. Their mutations suggest a possible polygenic inheritance of IA formation. Finally, our study will provide more informative and replicable causal susceptibilities to IA including four mutations in the second stage GWAS.

\section{Declarations}

\section{Data and resource availability}


The data that support the findings of this study was submitted as online supplemental material, and further detailed information is available upon request to the corresponding author. All genotype and phenotype resources are managed by "The First Korean Stroke Genetics Association Research" study constructed from the Sacred Heart Hospital Stroke Database.

\section{Acknowledgements}

This research is supported by the grant of the Korea Health Technology R\&D Project through the Korea Health Industry Development Institute (KHIDI), funded by the Ministry of Health \& Welfare, Republic of Korea (grant number: HR21C0198) and Hallym Research Fund.

\section{Author contributions}

E.P.H. and J.P.J. designed and managed this study. Analysis was done by E.P.H. and B.J.K Sample preparation and data collection were done by D.H.Y., B.J.K., E.P.H. and J.P.J. Drafting and reviewing of manuscript were done by E.P.H., D.H.Y., B.J.K., J.H.A., J.J.P., J.K.R. and J.P.J.

\section{Competing interests}

The authors declare no competing interests.

\section{References}

1. Tromp, G., Weinsheimer, S., Ronkainen, A. \& Kuivaniemi, H. Molecular basis and genetic predisposition to intracranial aneurysm. Ann. Med. 46, 597-606 (2014).

2. Asaithambi, G., Adil, M.M., Chaudhry, S.A. \& Qureshi, A.I. Incidences of unruptured intracranial aneurysms and subarachnoid hemorrhage: Results of a statewide study. J. Vasc. Interv. Neurol. 7, 14-17 (2014).

3. Zhou, S., Dion, P.A. \& Rouleau, G.A. Genetics of intracranial aneurysms. Stroke. 49, 780-787 (2018).

4. Vlak MH, Algra A, Brandenburg R, Rinkel GJ. Prevalence of unruptured intracranial aneurysms, with emphasis on sex, age, comorbidity, country, and time period: A systematic review and meta-analysis. Lancet. Neurol. 10, 626-636 (2011).

5. Boehme AK, Esenwa C, Elkind MS. Stroke risk factors, genetics, and prevention. Circ. Res. 120, 472-495 (2017).

6. Jeon, J.S. et al. A retrospective analysis on the natural history of incidental small paraclinoid unruptured aneurysm. J. Neurol. Neurosurg. Psychiatry. 85, 289-294 (2014).

7. Hackenberg, K.A.M., Hanggi, D. \& Etminan, N. Unruptured intracranial aneurysms. Stroke. 49, 2268-2275 (2018).

8. Freebern, E. et al. Gwas and fine-mapping of livability and six disease traits in holstein cattle. BMC. Genomics. 21, 41; 10.1186/s12864-020-6461-z (2020).

9. Schaid, D.J., Chen, W. \& Larson, N.B. From genome-wide associations to candidate causal variants by statistical fine-mapping. Nat. Rev. Genet. 19, 491-504 (2018).

10. Benner, C., Havulinna, A.S., Jarvelin, M.R., Salomaa, V., Ripatti, S. \& Pirinen, M. Prospects of fine-mapping trait-associated genomic regions by using summary statistics from genome-wide association studies. Am. J. Hum. Genet. 101, 539-551 (2017).

11. Sekar, A. et al. Schizophrenia risk from complex variation of complement component 4. Nature. 530, 177-183 (2016).

12. Hong, E.P. et al. Genomic variations in susceptibility to intracranial aneurysm in the korean population. J. Clin. Med. 8, 275; doi: 10.3390/jcm8020275 (2019).

13. Kim, B.J. et al. Genome-wide blood DNA methylation analysis in patients with delayed cerebral ischemia after subarachnoid hemorrhage. Sci. Rep. 10, 11419; doi: 10.1038/s41598-020-68325-3. (2020).

14. Benner, C., Spencer, C.C., Havulinna, A.S., Salomaa, V., Ripatti, S. \& Pirinen, M. Finemap: Efficient variable selection using summary data from genome-wide association studies. Bioinformatics. 32, 1493-1501 (2016).

15. Chang, C.C. et al. Second-generation plink: Rising to the challenge of larger and richer datasets. Gigascience. 4, 7; 10.1186/s13742015-0047-8 (2015).

16. Pruim, R.J. et al. Locuszoom: Regional visualization of genome-wide association scan results. Bioinformatics. 26, 2336-2337 (2010).

17. Wang, K., Li, M. \& Hakonarson, H. ANNOVAR: functional annotation of genetic variants from high-throughput sequencing data. Nucleic. Acids. Res. 38, e164; doi: 10.1093/nar/gkq603 (2010). 
18. GTEx Consortium. Genetic effects on gene expression across human tissues. Nature. 550, 204-213 (2017).

19. Warde-Farley, D. et al. The genemania prediction server: Biological network integration for gene prioritization and predicting gene function. Nucleic. Acids. Res. 38, W214-220 (2010).

20. Tam, V. et al. Benefits and limitations of genome-wide association studies. Nat. Rev. Genet. 20, 467-484 (2019).

21. Lohmueller, K.E., Pearce, C.L., Pike, M., Lander, E.S. \& Hirschhorn, J.N. Meta-analysis of genetic association studies supports a contribution of common variants to susceptibility to common disease. Nat Genet. 2003;33:177-182

22. Mata IF, Leverenz JB, Weintraub D, Trojanowski JQ, Chen-Plotkin A, Van Deerlin VM, et al. Gba variants are associated with a distinct pattern of cognitive deficits in parkinson's disease. Mov. Disord. 31, 95-102 (2016).

23. Beutler, E., Gelbart, T. \& Scott, C.R. Hematologically important mutations: Gaucher disease. Blood Cells Mol. Dis. 35, 355-364 (2005).

24. Kleinloog, R. et al. Rna sequencing analysis of intracranial aneurysm walls reveals involvement of lysosomes and immunoglobulins in rupture. Stroke. 47, 1286-1293 (2016).

25. Dee, R.A., Bai, X., Mack, C.P. \& Taylor, J.M. Molecular regulation of the rhogap graf3 and its capacity to limit blood pressure in vivo. Cells. 9, 1042; doi: 10.3390/cells9041042 (2020).

26. Bai. X. et al. The smooth muscle-selective rhogap graf3 is a critical regulator of vascular tone and hypertension. Nat. Commun. 4, 2910; doi: 10.1038/ncomms3910 (2013).

27. Inci, S. \& Spetzler, R.F. Intracranial aneurysms and arterial hypertension: A review and hypothesis. Surg. Neurol. 53, 530-540; discussion 540-532 (2000).

28. Oji, Y. et al. Variants in saposin d domain of prosaposin gene linked to parkinson's disease. Brain. 143, 1190-1205 (2020).

29. Robak, L.A. et al. Excessive burden of lysosomal storage disorder gene variants in parkinson's disease. Brain. 140, 3191-3203 (2017).

30. Wolinsky, H., Goldfischer, S., Daly, M.M., Kasak, L.E. \& Coltoff-Schiller, B. Arterial lysosomes and connective tissue in primate atherosclerosis and hypertension. Circ. Res. 36, 553-561 (1975).

31. Jerome, W.G. Lysosomes, cholesterol and atherosclerosis. Clin. Lipidol. 5, 853-865 (2010).

32. Hokari, M. et al. The impact of atherosclerotic factors on cerebral aneurysm is location dependent: Aneurysms in stroke patients and healthy controls. J. Stroke Cerebrovasc. Dis. 23, 2301-2307 (2014).

33. Song, T.J., Oh, S.H. \& Kim, J. The impact of statin therapy after surgical or endovascular treatment of cerebral aneurysms. J. Neurosurg. 1-8; doi: 10.3171/2019.3.JNS183500 (2019).

34. Wu, H. et al. The autophagy-lysosomal system in subarachnoid haemorrhage. J. Cell .Mol. Med. 20, 1770-1778 (2016).

35. Fernandez-Rhodes, L. et al. Importance of genetic studies of cardiometabolic disease in diverse populations. Circ. Res. 126, 18161840 (2020).

\section{Figures}



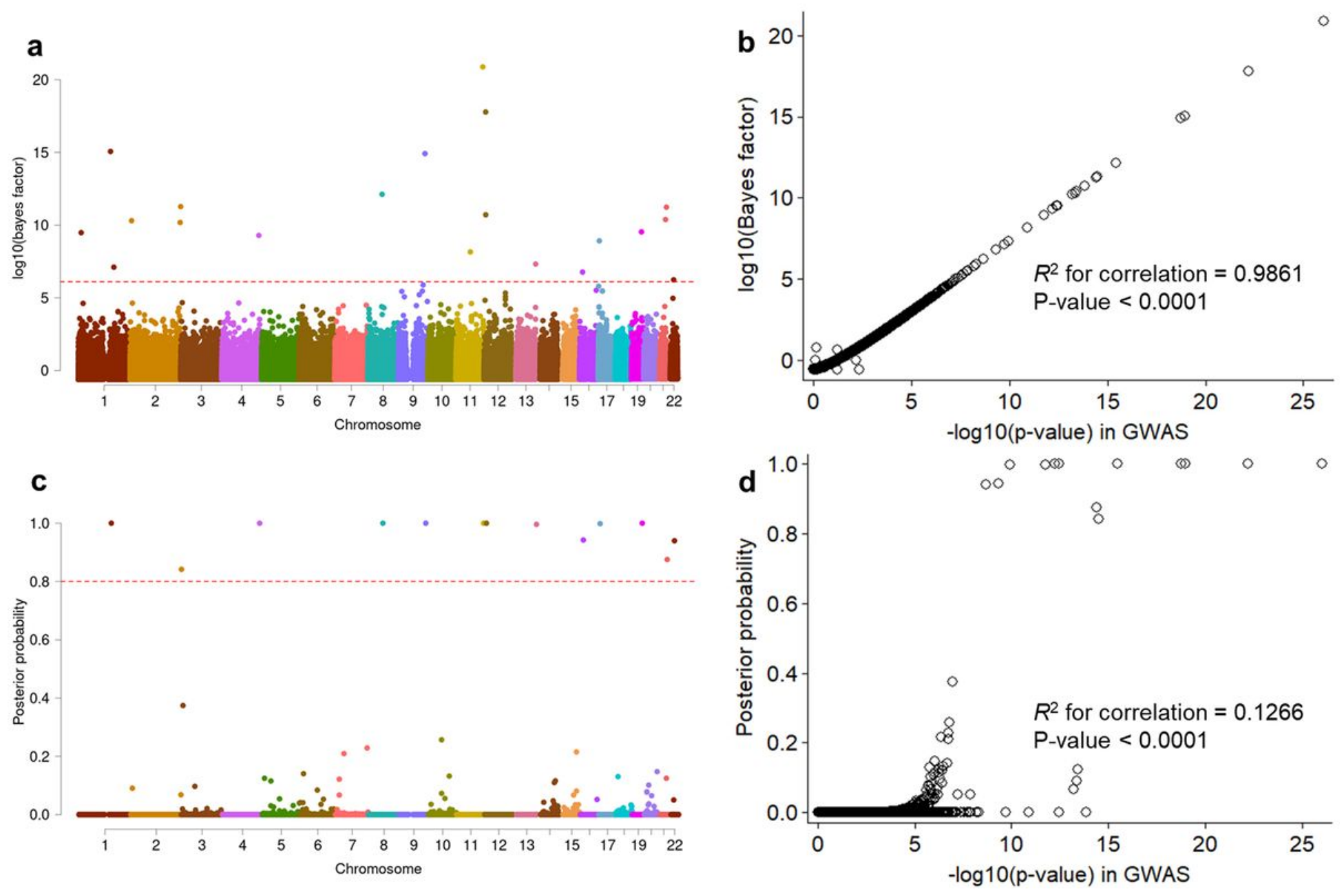

Figure 1

(a and c) Manhattan plots show log10-transformed Bayes factors (log10BF) and posterior inclusion probability (PIP) of variant causality estimations based on the summary statistics of a genome-wide association study (GWAS) of intracranial aneurysm (IA). (b and d) Plots compare the significance of IA GWAS, log10BF, and PIP. A red dash line indicates a genome-wide significance and a strong PIP of IA formation in the panels $A$ and $C$, respectively $(\log 10 B F=6.1$ and PIP $=0.8)$. R-square (R2) indicates the correlation between IA GWA p-value (-log10 transformed) and log10BF in panel B and PIP in panel D: P-value for R2. 

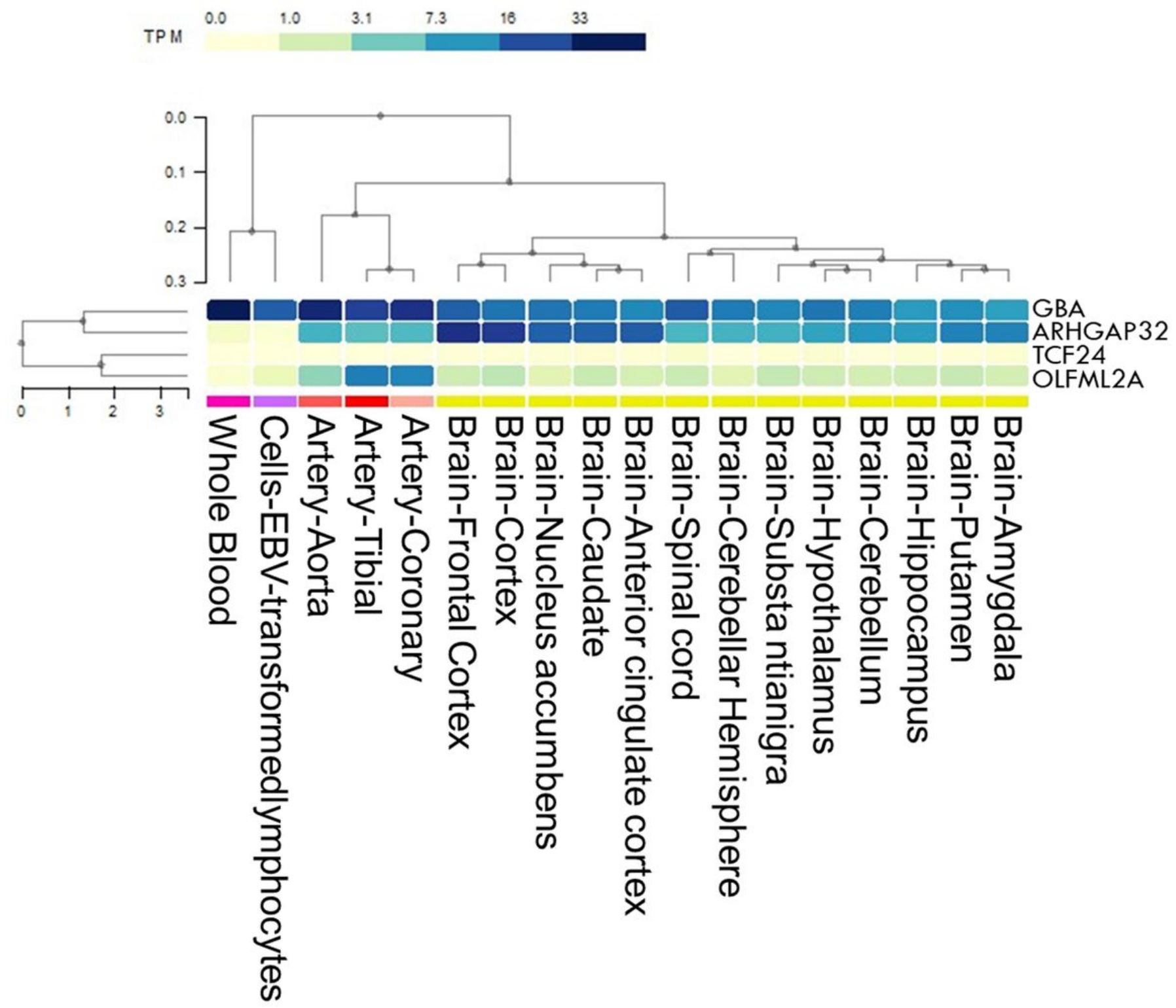

Figure 2

A heatmap of multiple gene expression involving GBA, TCF24, OLFML2A, and ARHGAP32 in human cells and tissues including artery, brain, and whole blood is presented. Gene expression was estimated as transcripts per million (TPM). Genes and types of cells or tissues were ordered via agglomerative hierarchical clustering. 


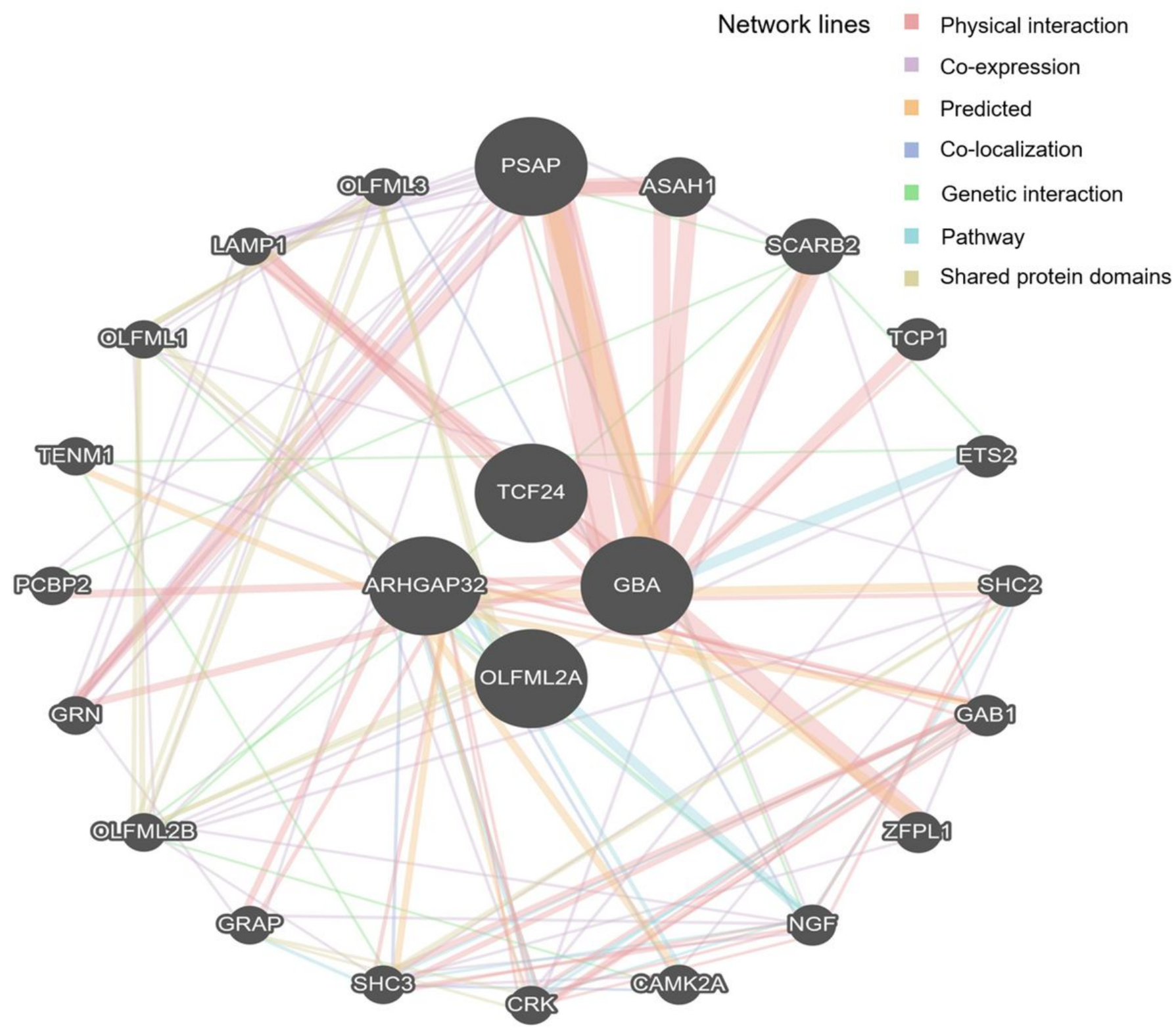

Figure 3

Susceptibility to intracranial aneurysm (Homo sapiens) based on multiple protein interactions between proteins coded by four causal hub genes including GBA, TCF24, OLFML2A, and ARHGAP32. The network included neighboring genes correlated with four hub genes. The width of individual lines indicates the intensity of the interaction between proteins. The colors in each line indicate multiple functions including physical interaction, co-expression, prediction, co-localization, genetic interaction, pathways, and shared protein domains.

\section{Supplementary Files}

This is a list of supplementary files associated with this preprint. Click to download.

- OnlineSupplementalData.docx 